Navaridas Nalda, F. y Jiménez Trens, M. A. (2016). Concepciones de los estudiantes sobre la eficacia de lós ambientes de aprendizaje universitarios. Revista de Investigación Educativa, 34(2), 503-519.

DOI: http://dx.doi.org/10.6018/rie.34.2.239481

\title{
Concepciones de los estudiantes sobre la eficacia de los ambientes de aprendizaje universitarios
}

\author{
Student' perceptions of the effectiveness \\ of university learning environments
}

Fermín Navaridas Nalda y María Asunción Jiménez Trens

Departamento de Ciencias de la Educación, Universidad de La Rioja. España

\begin{abstract}
Resumen
En este estudio se analizan las concepciones que tienen los estudiantes universitarios acerca de la utilidad o eficacia de los ambientes de aprendizaje que se contemplan con mayor frecuencia en las guías docentes de sus profesores. Para su realización se encuestó a 908 estudiantes matriculados en diferentes titulaciones de grado de la Universidad de La Rioja, todos ellos con edades comprendidas entre 18 y 22 años. Se utilizó un cuestionario de elaboración propia donde cada ambiente de aprendizaje seleccionado a modo de ítem admitía una doble respuesta, una referida a la autoeficacia percibida desde la propia experiencia personal como estudiante y otra relativa a la importancia que suponen que tiene el mismo ambiente para su profesores. Los resultados indican que el estudio individual y las explicaciones del profesor siguen siendo los ambientes más valorados por los estudiantes en términos de eficacia para el logro de los objetivos de aprendizaje universitario.

Palabras clave: concepciones de los estudiantes; ambientes de aprendizaje; eficacia percibida; enseñanza universitaria.
\end{abstract}

\begin{abstract}
This research analyses the perception that university students have regarding the usefulness and effectiveness of the different learning environments that are most frequently taken into account in the teaching guides of their professors. For the study, a total of 908 students enrolled

Correspondencia: Fermín Navaridas Nalda. E-mail: fermin.navaridas@unirioja.es. María Asunción Jiménez Trens. E-mail: asuncion.jtrens@unirioja.es. Departamento de Ciencias de la Educación. Universidad de La Rioja. España
\end{abstract}


on different degree courses at the University of La Rioja were surveyed, all of them aged between 18 and 22. A self-developed questionnaire was used where each of the teaching environments was as an item that could be answered twice: one referring to the students' perceived autoeffectiveness drawn from their personal experience as students; the other relative to the importance that students believe the same environment has for their professors. In terms of effectiveness, the results indicate that students assign the highest rating to individual study and professors' explanations as the best way to achieve their university learning objectives.

Keywords: student perception; learning environments; perceived effectiveness; Higher Education teachin.

\section{Introducción}

La amplia literatura a que está dando lugar el interés por la enseñanza y el aprendizaje en la Universidad ha generado resultados de investigación y modelos que permiten comprender mejor los aspectos que promueven una enseñanza y aprendizaje de calidad (Schommer-Aikins, Beuchat-Reichard y Hernández-Pina, 2012).

Calidad del aprendizaje que, aunque depende de factores personales y contextuales del estudiante, también está condicionada por los modelos de enseñanza del profesorado y por los ambientes de aprendizaje generados por esos modelos.

En este sentido, el paradigma de los enfoques de aprendizaje se ha revelado muy potente para mejorar la calidad del aprendizaje y para investigar el problema de la calidad de la educación superior (Hernández Pina, Arán Jara y Salmerón Pérez, 2012). Tales enfoques, se entienden como una combinación entre motivación y estrategia (Biggs, 1987; Monroy y Hernández Pina, 2014) y se producen como resultado de la interacción entre la percepción de la realidad contextual y las propias características del sujeto que aprende. Esta corriente, Student Approaches to Learning (SAL), analiza los mecanismos implicados en el aprendizaje, así como los factores personales y contextuales que influyen en tales enfoques. Se distinguen dos categorías de enfoques: profundo y superficial (Marton y Säljö, 1976), al que se ha añadido un tercero: estratégico (Entwistle y McCune, 2004). No obstante, estos enfoques no se presentan con carácter disyuntivo, sino que cada estudiante puede adoptar diversas formas o grados como si formaran parte de un continuo (Kember, 2000).

Además, los enfoques de aprendizaje se producen en unos contextos o ambientes de aprendizaje. Y estos ambientes, también, desempeñan un papel importante en el aprendizaje intencionado de los estudiantes (Baeten, Dochy, Struyven, Parmentier y Vanderbruggen, 2016; Lonka, Olkinuora y Mäkinen, 2004; López, Pérez-García y Rodríguez, 2015). Ambientes que abarcan no sólo métodos de enseñanza sino también las oportunidades que tiene el estudiante para aprender. Asimismo, el diseño de tales ambientes depende, en gran medida, de los modelos de actuación docente.

Por otro lado, la investigación sobre la enseñanza y el aprendizaje, desde la perspectiva fenomenológica, ha puesto de relieve la importancia de las concepciones. Algunos autores (Pratt, 1992; Thompson, 1992) las describen como ideas o creencias que se forman los profesores y estudiantes sobre el propio contexto educativo. En este sentido, también, la concepción que el profesorado tiene sobre la naturaleza del conocimiento que imparte influye en la configuración de los enfoques de aprendizaje de los estudiantes. Así, cuando 
el profesor manifiesta una concepción cuantitativa del conocimiento suele generar en el estudiante "aprender para reproducir (enfoque superficial)"; sin embargo, cuando esa concepción es predominantemente cualitativa, el efecto en el estudiante puede ser “aprender para comprender (enfoque profundo)" (Hernández Pina, 2002, p. 286).

Los ambientes de aprendizaje, también, están relacionados con las intenciones del profesor y las estrategias docentes que utiliza; a este respecto, se han identificado cinco modos diferentes de enfocar la enseñanza. Estas interpretaciones van desde aquellas centradas en el docente con la intención de transmitir información y basadas en la necesidad de dominio conceptual por parte del alumnado hasta aquellas centradas en el propio estudiante para que desarrolle sus propias concepciones, llegue a hacer suyo el saber y para que pueda cambiar sus concepciones acerca de los fenómenos estudiados (Hernández Pina, 2015; Kember, 1997; Prosser, Martin, Trigwell, Ramsden y Lueckenhausen, 2005; Trigwell, Prosser y Taylor, 1994).

De igual modo, estos ambientes tienen que ver con la interpretación que del aprendizaje hacen los estudiantes. Respecto al aprendizaje, se han reconocido seis concepciones que van desde aquellas que lo consideran como adquisición cuantitativa de conocimientos, memorización y adquisición de datos y procedimientos hasta la que se centra en el aprendizaje en cuanto a la producción del cambio como persona, pasando por aquellas que lo entienden como comprensión de significados y como proceso de interpretación de la realidad (Säljö; y Marton, como se citó en Hernández Pina, 2002, 2015).

Desde otra perspectiva, la investigación actual sobre aprendizaje está demostrando la capacidad de empoderamiento que éste tiene para el desarrollo de las personas y para la mejora de la calidad de vida (Hernández Pina, Martínez Clares, Martínez Juárez y Monroy Hernández, 2009). Quizás, el aprendizaje de calidad sea una de las principales herramientas con las que el ser humano pueda pertrecharse para afrontar la incertidumbre que el futuro le depare. De ahí, también, su relevancia.

\section{Revisión teórica sobre ambientes de aprendizaje universitarios}

Se constata la necesidad del profesorado de reflexionar, investigar y averiguar el modo como los conocimientos, mejor aún, las competencias son aprendidas por los estudiantes $y$, en consecuencia, el modo en que se puede enseñar eficazmente. Interés docente que trasciende la actualización científica, más patente aún desde la transformación promovida por el Espacio Europeo de Educación Superior (EEES; Hernández Pina et al, 2009). Un interesante análisis efectuado por Hernández Pina (2002), pone de manifiesto la relevancia del aprendizaje como puente entre docencia e investigación, que "podría tener implicaciones positivas para la mejora de la calidad institucional en su conjunto" (p. 296).

En coherencia, estas dos grandes cuestiones, enseñanza y aprendizaje en ámbito universitario, centran nuestro interés. A este respecto, cabe destacar una línea de investigación sobre el estudio de estrategias de enseñanza y aprendizaje universitario y, también, sobre concepciones de los profesores acerca del significado y viabilidad de la enseñanza y el aprendizaje (Hernández Pina y Maquilón Sánchez, 2010; Hernández Pina, Maquilón Sánchez, García Sanz y Monroy Hernández, 2010; Pintrich, Smith, García y McKeachie, 1991). 
Desde otro ángulo, el escenario del EEES ha evidenciado la necesidad de que los estudiantes desarrollen un aprendizaje autónomo y autorregulado como vía para alcanzar las competencias demandadas y para aumentar la calidad de su aprendizaje, lo que está promoviendo diferentes estudios (Hernández Pina, Rosário, Cuesta Sáez de Tejada, Martínez Clares y Ruiz Lara, 2006; Hernández Pina, Rosário y Cuesta Sáez de Tejada, 2010).

Una línea de investigación muy fecunda es la relativa a los enfoques del aprendizaje (Biggs, 1987; Entwistle, 1987/1988; Hernández Pina, Rodríguez Nieto, Ruiz Lara y Esquivel Cruz, 2010; Marton y Säljö, 1976; Ramsden, 1992, entre otros). Cabe destacar el trabajo de Monroy y Hernández Pina (2014), en el que se ponen de manifiesto factores influyentes en los enfoques de aprendizaje: motivación, conocimientos previos, concepciones de aprendizaje, creencias epistemológicas, cantidad de trabajo, percepción de los criterios de evaluación, concepciones y métodos de enseñanza de los profesores, titulación y tipo de tarea y contenido. Asimismo, se ha estudiado la relación entre enfoques de aprendizaje y rendimiento académico y afectivo: los estudiantes con enfoque profundo son los de mejores calificaciones y mayor satisfacción (Ruiz Lara, Hernández Pina y Ureña Villanueva, 2008).

Investigaciones sobre creencias epistemológicas han puesto de manifiesto la importancia de las mismas en la enseñanza, el aprendizaje y sus resultados. Así, cuando el estudiante tiene creencias no sofisticadas sobre el conocimiento (cierto, simple, manejable, dependiente de la autoridad) es más probable que simplifique la información aunque sea compleja; y, por el contrario, cuanto más sofisticadas sean (comprensión, flexibilidad en el pensamiento y la acción) los resultados suelen ser mejores. Recíprocamente, la enseñanza es factor relevante en la configuración de las creencias epistemológicas. En consecuencia, se aprecia una influencia mutua: las creencias influyen sobre los enfoques de aprendizaje y, de igual modo, determinadas experiencias educativas, tales como las que desarrollan un trabajo metacognitivo, contribuyen a modificar hacia una mayor sofisticación las propias creencias epistemológicas (Schommer-Aikins et al., 2012).

Un estudio realizado en Finlandia identifica los perfiles de estudiantes de la titulación de maestro, analizando la relación entre estrategias cognitivas y perfiles de estudiantes. Merece destacarse, entre las conclusiones, un mensaje importante para el profesorado universitario de maestros: en la creación de los ambientes de aprendizaje, los propios docentes deben manifestar cómo aprenden a regular su propio aprendizaje y deben ser capaces de mantener el soporte y ayuda para el aprendizaje de sus alumnos y fomentar sus habilidades de autorregulación (Heikkilä, Lonka, Nieminen y Niemivirta, 2012).

Con el fin de conocer la influencia de las metodologías de enseñanza universitaria sobre el alumnado y, dada la importancia de disponer de instrumentos adecuados para explorar los enfoques de enseñanza del profesorado universitario, Monroy, GonzálezGeraldo y Hernández-Pina (2015) han realizado una versión española (S-ATI-20) del Approaches to Teaching Inventory (ATI) (Trigwell y Prosser, 2004) eficaz para la autoreflexión y auto-evaluación del profesorado.

Analizar el efecto de distintas prácticas docentes en los resultados de los estudiantes, ha sido objeto de un trabajo, relacionando bases de datos internacionales (TALIS y PISA) que, aunque en ámbito de educación no universitaria, resulta inspirador para 
la educación superior en general (Méndez Martínez, 2015). En el caso concreto del contexto universitario, cabe destacar el estudio realizado por Gargallo López, Garfella Estéban, Sahuquillo Mateo, Verde Peleato y Jiménez Rodríguez (2015), quienes ponen de manifiesto la influencia de una metodología docente centrada en el estudiante en la mejora de la calidad del aprendizaje.

Respecto a las preferencias de los estudiantes por ambientes de aprendizaje universitarios, ámbito en el que se encuadra nuestro estudio, cabe destacar el trabajo de Baeten et al. (2016). Distinguen tres tipos de estudiantes, según si prefieren ambientes centrados: (a) en el profesor; (b) en el estudiante; (c) en ambos. También identifican preferencias de enseñanza y enfoques de aprendizaje, averiguando las relaciones entre ambos. Resulta llamativa la preferencia, en primer lugar, por la enseñanza directiva, guiada por el profesor. Menos sorprendente parecen las preferencias enfocadas al aprendizaje constructivo y al cooperativo, siendo la menos deseada aquella que genera aprendizaje pasivo. Acerca de las relaciones entre preferencias de enseñanza y enfoques de aprendizaje se averiguó que los estudiantes con enfoque profundo preferían enseñanza promotora del aprendizaje constructivo y cooperativo; por el contrario, los de enfoque superficial mostraron preferencias opuestas a los de enfoque profundo.

Partiendo de esta revisión, el presente trabajo se centra en las concepciones sobre los ambientes de aprendizaje. Consideramos ambientes de aprendizaje a los contextos interactivos en los que se produce el aprendizaje, como consecuencia de diferentes métodos de enseñanza (Baeten et al., 2016; Duarte, 2003). Tales ambientes representan una estructura que posibilita, mediante la docencia como uno de los factores influyentes, los diferentes enfoques de aprendizaje y logros de los estudiantes. Así, los estudiantes afrontan sus tareas según el modelado que se ejerce desde las estrategias de enseñanza y evaluación y que les incitan a modos diferentes de aprender. En síntesis, estos ambientes integran en una estructura la interacción entre el docente, que diseña y desarrolla su enseñanza conforme a sus creencias epistemológicas y pedagógico- didácticas y los estudiantes que tratan de aprender según sus percepciones de la enseñanza y sus enfoques de aprendizaje, en un determinado contexto educativo.

En consecuencia, el propósito de este trabajo consiste en una aproximación a las concepciones de los estudiantes sobre la eficacia de los ambientes de aprendizaje universitarios, diseñados por sus profesores en las correspondientes guías docentes. Se concreta como sigue a continuación.

\section{Objetivos}

1. Identificar las concepciones de los estudiantes universitarios respecto a la utilidad o eficacia de los ambientes de aprendizaje señalados con mayor frecuencia en las guías docentes universitarias.

2. Conocer la percepción de los estudiantes sobre la supuesta opinión de sus profesores acerca de la eficacia de los diferentes ambientes de aprendizaje.

3. Analizar las relaciones entre la eficacia atribuida por los estudiantes a los ambientes de aprendizaje y las percepciones de los estudiantes sobre la opinión de sus profesores acerca de la eficacia de los mismos ambientes. 


\section{Método}

\section{Diseño}

Se trata de un estudio de carácter descriptivo y exploratorio, mediante método de encuesta como procedimiento adecuado para recoger de forma rápida y precisa las concepciones de los estudiantes. Para ello, se elaboró un cuestionario ad hoc en el que cada ambiente de aprendizaje seleccionado a modo de ítem admitía una doble respuesta, una referida a la autoeficacia percibida desde la propia experiencia como estudiante y otra a la importancia que suponen que tiene el mismo ambiente para su profesores.

\section{Población y Muestra}

Durante el trabajo de campo se encuestó a 908 estudiantes matriculados en diferentes titulaciones y cursos de grado en la Universidad de La Rioja (España), lo que supone el $25,30 \%$ de la población investigada $(\mathrm{N}=3.589)$.

La muestra está formada por un 58,3\% (529) de mujeres frente al 41,6\% (379) de hombres, siendo la media de edad para ambos grupos de 21,39 años.

\section{Procedimiento de recogida de información}

El procedimiento de recogida de información se realizó mediante un cuestionario ad hoc anónimo, donde se pedía a los estudiantes que indicaran su opinión sobre la eficacia de una lista de diversos ambientes de aprendizaje utilizados durante la práctica docente universitaria: método expositivo, trabajos de investigación, ejercicios en grupo durante las clases, ejercicios individuales durante las clases, estudio individual, estudio acompañado fuera del aula, sistemas e-Learning. La selección de estos ambientes se efectuó teniendo en cuenta la frecuencia de uso en las guías docentes publicadas en el contexto universitario investigado.

Con el fin de garantizar la validez de contenido, se procedió a la valoración del cuestionario por parte de cuatro jueces expertos en docencia universitaria, acerca del grado en que el contenido del instrumento podía considerarse relevante y pertinente. Tomando en consideración las valoraciones efectuadas en relación con el diseño, la claridad y la comprensión de los ítems, se elaboró el cuestionario definitivo. La fiabilidad del cuestionario, estimada como consistencia interna a partir del alpha de Cronbach, ha sido de 0,81 .

Como puede observarse (Tabla 1), cada ambiente admitía una doble respuesta mediante dos escalas tipo Likert de 5 puntos, desde nula (1) a muchísima eficacia (5). Para la consecución de los objetivos, por una parte se preguntaba a los estudiantes por la preferencia personal con relación a cada uno de los ambientes de aprendizaje $\mathrm{y}$, por otro lado, la preferencia esperada en sus profesores con respecto a los mismos ambientes.

La administración del cuestionario se efectuó respetando criterios de anonimato, participación voluntaria y conocimiento del propósito de la investigación. 


\section{Tabla 1}

\section{Cuestionario sobre ambientes de aprendizaje}

\section{Instrucciones:}

Te presentamos una lista de diversos ambientes de aprendizaje. En tu opinión personal: 1) ¿Cuál es la utilidad, eficacia, de cada uno de ellos? 2) ¿Cuál es el valor que tú supones que van a darle tus profesores?

Es verdad que la eficacia puede variar de asignatura a asignatura, según el profesor; tú pon la media (tu impresión general).

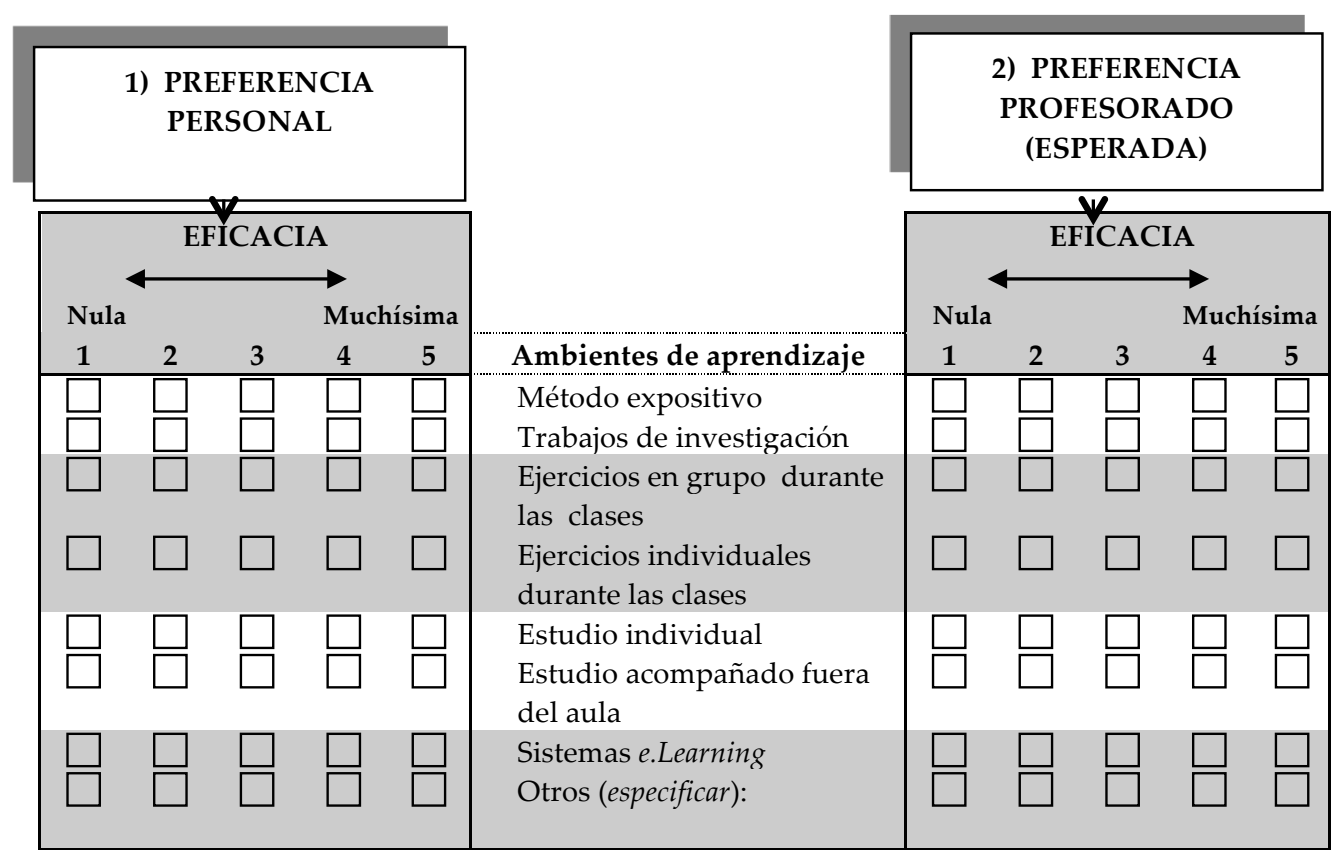

\section{Análisis de datos}

El análisis de datos se realizó mediante el paquete estadístico SPSS (versión 19.0 para Windows). Respecto a los dos primeros objetivos, para analizar las opiniones de los estudiantes, se efectuó el cálculo de estadísticos descriptivos como frecuencias, medias y desviaciones típicas. Además, para dar respuesta al tercer objetivo, se realizó un estudio comparado de las opiniones y percepciones antes descritas. En este sentido, atendiendo a la naturaleza ordinal de las variables, aplicamos la Prueba no paramétrica U de Mann-Whitney para analizar las diferencias entre las puntuaciones medias obtenidas. Posteriormente, a través de la Tau-b de Kendall analizamos las relaciones entre las dos distribuciones de puntuaciones emitidas y, más concretamente, el grado de coincidencia entre el orden de preferencia personal de los estudiantes y el orden de preferencia esperado en sus profesores. 


\section{Resultados}

Concepciones de los estudiantes universitarios de la eficacia de los ambientes de aprendizaje señalados con mayor frecuencia en las guías docentes universitarias

Respecto al primer objetivo, los resultados (Tabla 2) indican que los alumnos consideran que lo más eficaz es estudiar individuamente $(X=4,35)$ y atender las explicaciones del profesor $(X=3,91)$; representan, por tanto, una preferencia por ambientes centrados en los contenidos y en el profesor. Confirman, en parte, los resultados obtenidos por Cid Sabucedo, Pérez Abellás y Zabalza Beraza (2013), que encuentran, entre las prácticas docentes mejor valoradas por los estudiantes, aquellas consideradas propias de un "enfoque tradicional de la enseñanza". Asimismo, Baeten et al. (2016), identifican preferencias por la enseñanza guiada, directiva, interpretando que esto puede deberse a las prácticas a las que los estudiantes están habituados. Aunque ésta no sea la mejor alternativa (López et al., 2015). Seguidamente, y con diferencias no apreciables, se observa una valoración parecida de trabajos de investigación, ejercicios individuales en clase y estudio acompañado fuera del aula. Por último, los dos ambientes menos valorados son ejercicios en grupo durante las clases $(X=3,17)$ y sistemas e-Learning $(X=2,69)$. Curiosamente, esta concepción sobre la eficacia de los entornos de aprendizaje virtual confirma, en parte, resultados obtenidos en estudios recientes de la Organización para la Cooperación y el Desarrollo Económico (OCDE, 2015), donde se pone de manifiesto que las Tecnologías de la Información y la Comunicación (TIC) per se no tienen un efecto claro sobre el rendimiento académico.

Tabla 2

Preferencias de ambientes de aprendizaje

\begin{tabular}{lc}
\hline \multicolumn{2}{c}{ Concepciones personales } \\
\hline Ambientes de aprendizaje & Puntuaciones medias \\
\hline Estudio individual & 4,35 \\
Explicaciones del profesor & 3,91 \\
Trabajos de investigación & 3,38 \\
Ejercicios individuales durante clases & 3,37 \\
Estudio acompañado fuera del aula & 3,27 \\
Ejercicios en grupo durante clases & 3,17 \\
Sistemas e.Learning & 2,69 \\
\hline
\end{tabular}

Percepciones de los estudiantes sobre la supuesta opinión de sus profesores de la eficacia de los ambientes de aprendizaje

Respecto al segundo objetivo, los estudiantes perciben que los profesores, al igual que ellos mismos, consideran (Tabla 3) que el estudio individual $(X=4,17)$ y las explicaciones del profesor $(4,00)$ son los mejores ambientes de aprendizaje. Por el contrario, los modos de aprender menos útiles para los profesores, según sus alumnos, son los 
sistemas e.Learning $(X=3,00)$ y el estudio acompañado fuera del aula $(X=2,96)$. Atendiendo a las aportaciones de Kember (1997) sobre concepciones de la enseñanza, estos resultados nos permiten presumir la prevalencia de un ambiente de aprendizaje centrado en el profesor, con la intención de que los estudiantes adquieran de forma individual los conceptos más relevantes de la disciplina. Del mismo modo, es posible pensar, al igual que Baeten et al. (2016), que la eficacia percibida de los distintos ambientes de aprendizaje refleja en cierta medida el efecto que ejerce en los estudiantes las concepciones de la enseñanza universitaria predominante en sus profesores.

Tabla 3

Percepciones de los estudiantes sobre las supuestas preferencias docentes de ambientes de aprendizaje

\begin{tabular}{lc}
\hline Supuestas preferencias docentes de ambientes de aprendizaje & Puntuaciones medias \\
\hline Estudio individual & 4,17 \\
Explicaciones del profesor & 4,00 \\
Trabajos de investigación & 3,65 \\
Ejercicios individuales durante clases & 3,37 \\
Ejercicios en grupo durante clases & 3,08 \\
Sistemas e.Learning & 3,00 \\
Estudio acompañado fuera del aula & 2,96 \\
\hline
\end{tabular}

\section{Relaciones entre las preferencias personales de los estudiantes y las esperadas en sus profesores sobre la eficacia de los ambientes de aprendizaje}

El primer análisis comparativo entre las preferencias personales de los alumnos y las esperadas en sus profesores es a través de sus medias. El gráfico de perfiles (Figura 1) de las dos distribuciones de medias y los p-valores resultantes de los contrastes de medias para muestras pareadas permite destacar los siguientes resultados:

- Para seis de los siete ambientes de aprendizaje, existen diferencias estadísticamente significativas.

- Los alumnos, en términos generales, piensan que los profesores tienen una opinión coincidente con la suya respecto al ambiente centrado en ejercicios individuales durante las clases, con una puntuación media en eficacia de 3,37.

- Las mayores diferencias significativas positivas (opinión de los alumnos superior a la que creen que tienen los profesores) se aprecian en situaciones de estudio acompañado fuera del aula y estudio individual,

- Las mayores diferencias significativas negativas (opinión de los alumnos inferior a la que creen que tienen los profesores) se observan en los ambientes centrados en los trabajos de investigación y sistemas e.Learning.

- Finalmente, y para los dos ambientes mejor puntuados, cabe pensar en cierto corporativismo entre grupos: los alumnos prefieren el estudio individual, y consideran que los profesores valoran más las explicaciones docentes $(4,35$ y 3,9, alumnos, frente a 4,17 y 4,00, profesores). 


\begin{tabular}{|c|c|c|c|c|}
\hline Ambientes de aprendizaje & 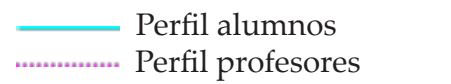 & $\begin{array}{c}X \\
\text { Alum }\end{array}$ & $\begin{array}{c}X \\
\text { Profes }\end{array}$ & $\begin{array}{l}\text { Sig. } \\
\text { p-val }\end{array}$ \\
\hline Estudio individual & & 4,35 & 4,17 & 0,000 \\
\hline Explicaciones del profesor & & 3,91 & 4,00 & 0,031 \\
\hline Trabajos de investigación & & 3,38 & 3,65 & 0,000 \\
\hline Ejercicios individuales en clase & & 3,37 & 3,37 & 0,533 \\
\hline Estudio acompañado fuera del aula & & 3,27 & 2,96 & 0,000 \\
\hline Ejercicios en grupo durante clases & & 3,17 & 3,08 & 0,037 \\
\hline Sistemas e.Learning & $i$ & 2,69 & 3,00 & 0,000 \\
\hline
\end{tabular}

Figura 1. Valoraciones medias sobre la eficacia de ambientes de aprendizaje, de los estudiantes acerca de sí mismos y de la concepción que atribuyen a los profesores.

Estos resultados, así como los de Baeten et al. (2016) y Lonka et al. (2004), entre otros, invitan a realizar un siguiente nivel de análisis para comprobar si existe relación entre las dos distribuciones de puntuaciones. Para ello optamos por aplicar una medida apropiada para variables ordinales, en concreto la Tau de Kendall. Esta medida, al igual que el coeficiente de correlación de Pearson, toma valores en el intervalo $[-1,+1]$ tal que valores próximos a 1 indican fuerte asociación positiva (a medida que aumentan los valores de una variable, aumentan los valores de la otra) y valores próximos a -1 indican fuerte asociación negativa (a medida que aumentan los valores de una variable, disminuyen los de la otra).

De las dos versiones de la Tau de Kendall, Tau-b aconsejable para variables con el mismo número de categorías y Tau-c válida para variables con distinto número de categorías aunque tiende a subestimar el verdadero grado de asociación, aplicamos la primera. De los resultados (Tabla 4) puede avanzarse una primera conclusión: la relación entre las dos variables es positiva $y$, aunque significativa al $1 \%$ en seis de los

Tabla 4

Nivel de asociación entre el orden de preferencia personal de los estudiantes y el orden de preferencia esperado en sus profesores sobre ambientes de aprendizaje

\begin{tabular}{lcc}
\hline Ambientes de aprendizaje & Tau-b de Kendall & p-valor \\
\hline Estudio individual & 0.315 & 0.000 \\
Explicaciones del profesor & 0.072 & 0.014 \\
Trabajos de investigación & 0.187 & 0.000 \\
Ejercicios individuales durante clases & 0.229 & 0.000 \\
Estudio acompañado fuera del aula & 0.287 & 0.000 \\
Ejercicios en grupo durante clases & 0.171 & 0.000 \\
Sistemas e.Learning & 0.292 & 0.000 \\
\hline
\end{tabular}


siete ambientes y al 5\% en el restante, bastante reducida. El nivel de asociación más alto se da en el estudio individual $(0,315)$ y el más bajo en las explicaciones del profesor $(0,072)$. Así con todo, parece probarse que la concepción que tienen los estudiantes sobre la eficacia de los ambientes de aprendizaje guarda cierta relación con la idea previa que se forman de lo que el contexto docente les exige y les ofrece (Biggs, 2006).

Por último, y con el fin de indagar sobre los desacuerdos, creamos la variable Preferencias personales-Preferencias profesores esperadas, que denominamos variable grado de discrepancia en el proceso de aprendizaje-enseñanza (D), tomando valores de -4, a 4. Los resultados más relevantes del análisis realizado son los que siguen (Tabla 5):

- El mayor grado de coincidencia $(D=0)$ se produce en el caso de los sistemas e.Learning, con un $40 \%$ de las valoraciones.

- El menor grado de coincidencia se da en el estudio individual, 25\%, siendo las discrepancias fundamentalmente de signo positivo $(D=1,2,3,4), 64 \%$ frente a un $11 \%$ de negativas.

- Las discrepancias positivas son también bastante más frecuentes que las negativas $(\mathrm{D}=-1,-2,-3,-4)$ en el ambiente centrado en el estudio acompañado fuera del aula, $42 \%$ frente a $24 \%$.

- Por último y en lo que respecta a las discrepancias de signo negativo, destaca el caso de los trabajos de investigación, con el $49 \%$ frente al $22 \%$ de signo positivo; también sobresale en el mismo sentido el sistema e.Learning (40\% son negativas frente al $20 \%$ de positivas).

Tabla 5

Grado de discrepancia entre preferencias de ambientes de los estudiantes y preferencias esperadas en sus profesores.

\begin{tabular}{lccccc}
\hline \multirow{2}{*}{ Ambientes de aprendizaje } & \multicolumn{5}{c}{ Grado de discrepancia, D: frecuencias relativas en \% } \\
\cline { 2 - 6 } & $\mathbf{D}=\mathbf{0}$ & $\mathbf{D}=\mathbf{\pm}$ & $\mathbf{D}> \pm \mathbf{1}$ & $\mathbf{D}$ positivas & D negativas \\
\hline Estudio individual & 25 & 37 & 38 & 64 & 11 \\
Explicaciones del profesor & 34 & 43 & 23 & 28 & 38 \\
Trabajos de investigación & 29 & 45 & 26 & 22 & 49 \\
Ejercicios individuales durante clases & 38 & 44 & 18 & 32 & 30 \\
Estudio acompañado fuera del aula & 34 & 43 & 23 & 42 & 24 \\
Ejercicios en grupo durante clases & 30 & 45 & 25 & 33 & 37 \\
Sistemas e.Learning & 40 & 38 & 22 & 20 & 40 \\
\hline
\end{tabular}

\section{Discusión y conclusiones}

Respecto al primer objetivo, las concepciones de los estudiantes investigados revelan claramente una preferencia por ambientes de aprendizaje centrados en el contenido y en la enseñanza expositiva, ya que valoran especialmente el estudio individual y las 
explicaciones del profesor. Se puede afirmar, por tanto, que se inclinan por ambientes dirigidos por el profesor en lugar de hacerlo por aquellos que otorgan mayor protagonismo al estudiante. Asimismo, sorprende que prácticas educativas consideradas más innovadoras y necesarias en el contexto del EEES, como son: trabajos de investigación, estudio acompañado fuera del aula o sistemas e-Learning, sean bastante menos valoradas. Estos resultados coinciden, en parte, con los obtenidos por Baeten et al. (2016) en un contexto similar de investigación, destacando también en su caso una mayor preferencia por la enseñanza guiada por el profesor. De todos modos, es preciso matizar que, tal como acreditan los autores citados, estas preferencias no han sido confirmadas en la totalidad de las investigaciones revisadas. En consecuencia, conocer tales preferencias "sigue siendo un área de investigación proactiva para explorar" (p. 4).

Centrándonos en la realidad educativa investigada, ¿a qué se debe que los estudiantes consideren que los ambientes más eficaces son el estudio individual y el método expositivo? Las razones precisas las desconocemos, debido al carácter descriptivo del presente trabajo. Sin embargo, cabe pensar en la posible relación entre las preferencias por ambientes centrados en el profesor y el enfoque de aprendizaje superficial; no obstante, este extremo no ha sido confirmado de manera inequívoca en todas las investigaciones (véase, por ejemplo, la revisión sistemática sobre factores influyentes en los enfoques de aprendizaje efectuada por Monroy y Hernández Pina, 2014). Asimismo, otra explicación puede radicar en el comportamiento estratégico de los estudiantes durante la actividad de estudio (Entwistle y McCune, 2004). Desde esta perspectiva, el enfoque adoptado por los estudiantes podría variar de superficial a profundo, y viceversa, en función de las exigencias docentes y de evaluación, más que por encontrar el verdadero sentido del aprendizaje. Respecto a la evaluación, son numerosos los estudios que muestran que la forma en que los estudiantes se acercan a su aprendizaje está fuertemente influenciada por la forma en que se evalúa (Baeten et al., 2016; Biggs, 1999/2006; Gargallo López et al., 2015; Monroy y Hernández Pina, 2014). De acuerdo con la importancia de esta idea, Hernández Pina et al. (2012) han estudiado la relación de metodologías de enseñanza y procedimientos evaluadores con los enfoques de aprendizaje de diferente intensidad y nivel de avance académico. Entre las conclusiones obtenidas, cabe destacar la que se refiere a la necesidad de generar estrategias de enseñanza activa y contextos de aprendizaje que faciliten y promuevan estrategias de aprendizaje dirigidas a estimular la predisposición a adoptar enfoque profundo de intensidad alta.

Además, el profesorado, como nos recuerdan Biggs (1999/2006) y Kember (1997, 2000), también influye en el diseño de los ambientes de aprendizaje mediante sus concepciones acerca de cómo desarrollar su docencia en el marco del EEES; y este modelo le exige atender al estudiante en su proceso de aprendizaje desde la orientación, la tutoría, el asesoramiento, fomentando su autonomía y la construcción social del conocimiento (López et al., 2015). Asimismo, hay que tener en cuenta que la educación universitaria ha de incorporar modelos de aprender que estimulen la comprensión y el uso estratégico del conocimiento, mediante actividades docentes que promuevan la reflexión y el cuestionamiento, que hagan posible el proceso de atribuir sentido personal al aprendizaje con predominio de la motivación intrínseca, para conseguir que los estudiantes adopten un enfoque profundo (Hernández Pina et al., 2012). 
Por otro lado, y atendiendo a la utilidad percibida por los estudiantes investigados en torno a sistemas e.Learning, nos cuestionamos si realmente los recursos tecnológicos emergentes están produciendo el cambio cualitativo e innovador esperado en los procesos de enseñanza y aprendizaje universitarios o si, por el contrario, su incorporación conlleva la mera reproducción de formas de hacer tradicionales sobre una nueva base tecnológica. En nuestra opinión, el reto educativo no consiste en digitalizar la enseñanza tradicional universitaria, sino más bien en hacer efectiva la integración pedagógica de los recursos tecnológicos en el currículum para la mejora de la calidad del aprendizaje. En esta misma línea de pensamiento, Lonka et al. (2004) nos advierten de nuevos ambientes de aprendizaje marcados por la necesidad del aprendizaje en colaboración y la gestión de la información proporcionada por las TIC que, posiblemente, requieran nuevos enfoques. Quizás por ello, el "enfoque profundo puede ser reevaluado en el nuevo ambiente de aprendizaje” (p. 308), ya que, según apuntan estos investigadores, este enfoque puede desembocar en un trabajo demasiado individualista, incompatible con la formación en competencias demandada por el Espacio Europeo de Educación Superior. En cualquier caso, parece necesario combinar diferentes ambientes de aprendizaje para dar adecuada respuesta a la diversidad de necesidades del alumnado.

En resumen, y en sintonía con otros autores (Vázquez García, 2015), entendemos que estos resultados pueden suscitar una significativa reflexión en el profesorado del contexto investigado acerca de la conveniencia o necesidad de desarrollar metodologías centradas en el estudiante, ya que parece demostrarse su efecto positivo en la mejora del clima motivacional y, en definitiva, sobre la calidad del aprendizaje, como ponen de manifiesto Gargallo López et al. (2015).

En relación al segundo objetivo, la percepción de los estudiantes acerca de lo que opinan sus profesores coincide, en términos generales, con sus preferencias personales. Congruentemente con el trabajo desarrollado por otros autores (Pratt, 1992; Thompson, 1992), este resultado nos hace pensar que la concepción que los estudiantes se forman de lo que el contexto instructivo les exige y les ofrece (actividad docente) influye sobre su modo de afrontar las tareas de aprendizaje. Desde esta interpretación, advertimos, al igual que Hernández Pina y Maquilón Sánchez (2010), que un enfoque de enseñanza centrado preferentemente en el profesor puede condicionar un trabajo del estudiante demasiado individualista, algo incompatible ante el reto universitario del aprendizaje de competencias para la formación integral.

Finalmente, en cuanto a las relaciones entre las concepciones de los estudiantes sobre la opinión de sus profesores de la eficacia de los ambientes y la propia eficacia percibida, parece probarse una cierta relación entre las preferencias personales de los estudiantes y las esperadas en los profesores. No obstante, los alumnos reconocen como más eficaz el trabajo individual que lo que perciben en la actividad docente de sus profesores, confirmando de este modo los resultados obtenidos en estudios anteriores en este mismo ámbito (Baeten et al., 2016; Cid Sabucedo et al., 2013; López et al., 2015). De modo coherente, consideran que sus profesores valoran más sus explicaciones que el estudio individual de los estudiantes. En este sentido, y como señalan Lonka et al. (2004), la investigación sobre estas cuestiones es relevante para comprender mejor el proceso de aprendizaje de los estudiantes. 
Sintetizando, la interpretación que hacen los estudiantes investigados sobre la eficacia de los ambientes de aprendizaje sitúa el foco central de atención en la adquisición de conocimientos, mediante el estudio individual y la explicación docente, que permiten dar respuestas acertadas al final del proceso formativo. En esta situación, el profesor adopta un papel relevante para la eficacia del proceso, creando el ambiente adecuado para facilitar la adquisición de los conceptos fundamentales de la disciplina. Así, sobre la base de otros trabajos (Biggs, 1999/2006; Prosser et al., 2005), cabe pensar que en la medida en que los estudiantes perciben que un ambiente de aprendizaje resulta útil para conseguir mejores calificaciones en una asignatura, adoptan un comportamiento concreto durante la práctica educativa.

En conclusión, estos resultados sugieren que las concepciones de los estudiantes sobre la eficacia de los ambientes de aprendizaje investigados se encuentran relacionadas con los enfoques de enseñanza que adoptan sus profesores. Sin embargo, debemos ser precavidos con esta interpretación, debido a las limitaciones que se derivan de un diseño de investigación de carácter descriptivo como el utilizado en este trabajo.

Por consiguiente, consideramos necesario ampliar esta vía de investigación iniciada, adoptando un nuevo diseño metodológico orientado a averiguar las posibles relaciones entre las preferencias de los estudiantes por diferentes ambientes de aprendizaje, los enfoques de aprendizaje adoptados y el rendimiento académico. Asimismo, otra línea de investigación puede centrarse en explorar si se producen cambios en el tránsito del enfoque superficial al profundo, como consecuencia de la aplicación de modelos de enseñanza centrados en los estudiantes. Entendemos que ello permitirá identificar factores que incrementan la calidad de la enseñanza universitaria ofertada.

\section{Referencias}

Baeten, M., Dochy, F., Struyven, K., Parmentier, E. y Vanderbruggen, A. (2016). Studentcentred learning environments: An investigation into student teacher's instructional preferences and approaches to learning. Learning Environments Research. 19(1), 43-62. doi: 10.1007/s10984-015-9190-5

Biggs, J. (1987). Student approaches to learning and studying. Hawthorn, Australia: Australian Council for Educational Research.

Biggs, J. (2006). Calidad del aprendizaje universitario (Trad. P. Manzano; 2a ed). Madrid: Narcea. (Reimpreso de Teaching for quality learning at university, por J. Biggs, Ed., 1999, Reino Unido: Open University Press).

Cid Sabucedo, A., Pérez Abellás, A. y Zabalza Beraza, M.A. (2013). Las prácticas de enseñanza realizadas/observadas de los "mejores profesores" de la Universidad de Vigo. Educación XXI, 16(2), 265-296. doi: 10.5944/educxx1.16.2.2643

Duarte, J. (2003). Ambientes de aprendizaje: Una aproximación conceptual. Estudios Pedagógicos, 29, 97-113. doi: 10.4067/S0718-07052003000100007

Entwistle, N.J. (1988). La comprensión del aprendizaje en el aula (Trad. Anónimo; Vol. 10). Barcelona: Paidós-MEC. (Reimpreso de Understanding classroom learning: Changing perspectives in education series, N.J.Entwistle, Ed., 1987, Reino Unido: Hodder \& Stoughton). 
Entwistle, N.J. y McCune, V. (2004). The conceptual bases of study strategy inventories. Educational PsyChology, 16(4), 325-346. Recuperado de www.jstor.org/stable/23363875 Gargallo López, B., Garfella Estéban, P., Sahuquillo Mateo, P., Verde Peleato, I. y Jiménez Rodríguez, M. A. (2015). Métodos centrados en el aprendizaje, estrategias y enfoques de aprendizaje en estudiantes universitarios. Revista de Educación, 370, 229-254. doi: 10.4438/1988-592X-RE-2015-370-304

Heikkilä, A., Lonka, K., Nieminen, J. y Niemivirta, M. (2012). Relations between teacher students'approaches to learning, cognitive and attributional strategies, well-being and study success. Higher Education, 64, 455-471. doi: 10.1007/s10734-012-9504-9

Hernández Pina, F. (2002). Docencia e investigación en educación superior. Revista de Investigación Educativa, 20(2), 271-301. Recuperado de http://revistas.um.es/rie/ article/view/98921

Hernández Pina, F. (26 de febrero de 2015). Factores de calidad en educación superior: Enseñanza, aprendizaje y competencias. Tres puntos clave en el docente [Material docente]. Plan de formación del PDI (Seminario de Formación del PDI 2014-2015. Procesos de enseñanza y aprendizaje). Universidad de la Rioja: La Rioja.

Hernández Pina, F., Arán Jara, A. y Salmerón Pérez, H. (2012). Enfoques de aprendizaje y metodologías de enseñanza en la Universidad. Revista Iberoamericana de Educación 60(3), 1-10. Recuperado de http://rieoei.org/deloslectores/4878Hdez.pdf

Hernández Pina, F. y Maquilón Sánchez, J.J. (2010). Las concepciones de la enseñanza. Aportaciones para la formación del profesorado. Revista Electrónica Universitaria de Formación del Profesorado, 13(3), 17-25. Recuperado de http://www.redalyc.org/ articulo.oa?id=217015214002

Hernández Pina, F., Maquilón Sánchez, J.J., García Sanz, M.P. y Monroy Hernández, F. (2010). Concepciones de la enseñanza y el aprendizaje en profesorado de educación superior. Psicología Educativa, 16(2), 95-105. Recuperado de https://dialnet.unirioja. es/ejemplar/255722

Hernández Pina, F., Martínez Clares, P., Martínez Juárez, M. y Monroy Hernández, F. (2009). Aprendizaje y competencias. Una nueva mirada. Revista Española de Orientación y Psicopedagogía, 20(3), 312-319. Recuperado de http://www.redalyc.org/articulo. oa?id=338230783009

Hernández Pina, F., Rodríguez Nieto, M.C., Ruiz Lara, E. y Esquivel Cruz, J.E. (2010). Enfoques de aprendizaje en alumnos universitarios de la titulación de Ciencias de la Actividad Física y del Deporte de España y México. Revista Iberoamericana de Educación, 53(7), 1-11. Recuperado de http://rieoei.org/3426.htm

Hernández-Pina, F., Rosário, P. y Cuesta Sáez de Tejada, J.D. (2010). Impacto de un programa de autorregulación del aprendizaje en estudiantes de Grado. Revista de Educación, 353, 571-588. Recuperado de http://www.revistaeducacion.mec.es/ re353_21.html

Hernández Pina, F., Rosário, P., Cuesta Sáez de Tejada, J.D., Martínez Clares, P. y Ruiz Lara, E. (2006). Promoción del aprendizaje estratégico y competencias de aprendizaje en estudiantes de primero de universidad: evaluación de una intervención. Revista de Investigación Educativa, 24(2), 615-631. Recuperado de http://revistas.um.es/rie/ article/view/97221 
Kember, D. (1997). A reconceptualisation of the research into university academics' conceptions of teaching. Learning and Instruction, 7(3), 255-275. doi: 10.1016/ S0959-4752(96)00028-X

Kember, D. (2000). Misconceptions about the learning approaches, motivation and study practices of Asian students. Higher Education, 40(1), 99-121. doi: 10.1023/A:10004036826490

Lonka, K., Olkinuora, E. y Mäkinen, J. (2004). Aspects and prospects of measuring studying and learning in higher education. Educational Psychology Review, 16(4), 301-323. doi: 10.1007/s10648-04-0002-1

López, M.C., Pérez-García, M.P. y Rodríguez, M.J. (2015). Concepciones del profesorado universitario sobre la formación en el marco del espacio europeo de educación superior. Revista de Investigación Educativa, 33(1), 179-194. doi: 10.6018/rie.33.1.189811

Marton, F. y Säljö, R. (1976). On qualitative differences in learning: I. Outcome and process. British Journal of Educational Psychology, 46, 4-11. doi: 10j.2044-8279.1976.tb02980.x

Méndez Martínez, I. (2015). Prácticas docentes y rendimiento estudiantil. Evidencia a partir de TALIS 2013 y PISA 2012. Logroño/Madrid: Consejería de Educación, Cultura y Turismo del Gobierno de La Rioja, Fundación Santillana, Instituto Nacional de Evaluación Educativa (INEE).

Monroy, F. y Hernández Pina, F. (2014). Factores que influyen en los enfoques de aprendizaje universitario. Una revisión sistemática. Educación XXI, 17(2), 105-124, doi: 10.5944/educxx1.17.2.11481

Monroy, F., González-Geraldo, J.L. y Hernández-Pina, F. (2015). A psychometric analysis of the approaches to teaching inventory (ATI) and a proposal for a Spanish version (S-ATI-20). Anales de Psicología, 31(1), 172-183. http://dx.doi.org/10.6018/ analesps.31.1.190261

Organización para la Cooperación y el Desarrollo Económico (2015). Students, Computers and Learning: Making the Connection (OCDE Publicación No. s.n.). doi: http://dx.doi.org/10.1787/9789264239555-en

Pintrich, P.R., Smith, D.A., García, T. y McKeachie, W.J. (1991). A manual for the use of the motivational strategies for learning questionnaire (MSLQ). Ann Arbord, EE.UU.: NCRIPTAL.

Pratt, D.D. (1992). Chinese conceptions of learning and teaching: a westerner's attempt at understanding. International Journal of Lifelong Education, 11(4), 301-319. doi: 10.1080/0260137920110404

Prosser, M., Martin, E., Trigwell, K., Ramsden, P. y Lueckenhausen, G. (2005). Academics' experiences of understanding of their subject matter and the relationship of this to their experience of teaching and learning. Instructional Science, 33, 137-157. doi: 10.1007/s11251-004-7687-x

Ruiz Lara, E., Hernández Pina, F. y Ureña Villanueva, F. (2008). Enfoques de aprendizaje y rendimiento institucional y afectivo de los alumnos de la titulación de Ciencias de la Actividad Física y del Deporte. Revista de Investigación Educativa, 26(2), 307-322. Recuperado de http://revistas.um.es/rie/article/view/93941

Schommer-Aikins, M., Beuchat-Reichardt, M. y Hernández-Pina, F. (2012). Creencias epistemológicas y de aprendizaje en la formación inicial de profesores. Anales de Psicología, 28(2), 465-474. doi: 10.6018/analesps.28.2.125341 
Thompson, A. G. (1992). Teachers' beliefs and conceptions: A synthesis of the research. En D.A. Grouws (Ed.), Handbook of research on mathematics teaching and learning. Nueva York, EE.UU.: Macmillan.

Trigwell, K., Prosser, M. y Taylor, P. (1994). Qualitative differences in approaches to teaching first year university sciences. Higher Education, 27(1), 75-84. doi: 10.1007/ BF01383761

Trigwell, K. y Prosser, M. (2004). Development and use of the approaches to teaching inventory. Educational Psychology Review, 16(4), 409-424. doi: 10.1007/s10648-0040007-9

Vázquez García, J.A. (2015). Nuevos escenarios y tendencias universitarias. Revista de Investigación Educativa, 33(1), 13-26. doi: http://dx.doi.org/10.6018/rie.33.1.211501

Fecha de recepción: 14 de octubre de 2015.

Fecha de revisión: 14 de octubre de 2015.

Fecha de aceptación: 29 de marzo de 2016. 
